\title{
Réponses de trois espèces sentinelles aquatiques à la contamination en métaux traces dans deux rivières de l'Abitibi, Québec
}

\section{Response of three aquatic sentinel species to metal trace contamination in two rivers in the Abitibi region, Québec}

\author{
S. Masson, P. G.C. Campbell, C. Olsen, R. Martel, B. Pinel-Alloul, B. Béthot et A. \\ Hontela
}

Volume 18, numéro hors-série, 2005

URI : https://id.erudit.org/iderudit/705581ar

DOI : https://doi.org/10.7202/705581ar

Aller au sommaire du numéro

Éditeur(s)

Université du Québec - INRS-Eau, Terre et Environnement (INRS-ETE)

ISSN

0992-7158 (imprimé)

1718-8598 (numérique)

Découvrir la revue

Citer cet article

Masson, S., Campbell, P. G., Olsen, C., Martel, R., Pinel-Alloul, B., Béthot, B. \& Hontela, A. (2005). Réponses de trois espèces sentinelles aquatiques à la contamination en métaux traces dans deux rivières de l'Abitibi, Québec. Revue des sciences de l'eau / Journal of Water Science, 18, 143-160.

https://doi.org/10.7202/705581ar

\section{Résumé de l'article}

La métallothionéine (MT) est un ligand protéinique intracellulaire important à cause de son implication dans les processus de régulation des métaux traces essentiels et non-essentiels. Bon nombre de travaux ont été conduits en laboratoire pour bien comprendre les mécanismes de synthèse de cette protéine, mais les études réalisées en milieu aquatique sont beaucoup plus rares, particulièrement dans les rivières (milieu lotique). Dans ce contexte, la présente étude avait deux objectifs principaux : (1) identifier en milieu lotique les métaux traces (argent "Ag", cadmium "Cd", cuivre "Cu", nickel "Ni", plomb "Pb" et zinc "Zn") susceptibles d'induire la synthèse de la MT chez trois espèces sentinelles, soit le mollusque bivalve Pyganodon grandis, la larve d'éphémère Hexagenia limbata et le grand brochet du nord Esox lucius ; (2) définir les variables physico-chimiques ou toxicologiques pouvant influencer la prise en charge des métaux traces et conséquemment les concentrations en MT chez le mollusque $\mathrm{P}$. grandis et l'éphémère $H$. limbata. Deux rivières de la région de l'Abitibi ont été échantillonnées à l'été 2001 afin de récolter les espèces sentinelles à 21 stations. Les concentrations en métaux traces, ainsi qu'en métallothionéine, ont été mesurées dans les branchies des mollusques, dans le rein des brochets et dans le corps entier des éphémères. La métallothionéine a été mesurée à l'aide d'une technique de saturation au mercure. D'après nos résultats, le cadmium tissulaire semble être le meilleur prédicteur de la concentration de MT chez les trois espèces sentinelles. Cependant, l'implication d'autres métaux est également possible. Dans le cas de $P$. grandis, la concentration de manganèse $(\mathrm{Mn})$ dans les sédiments pourrait influencer la prise en charge du Cd et la synthèse de MT. 


\title{
Réponses de trois espèces sentinelles aquatiques à la contamination en métaux traces dans deux rivières de l'Abitibi, Québec
}

\section{Response of three aquatic sentinel species to metal trace contamination in two rivers in the Abitibi region, Québec}

\author{
S. MASSON ${ }^{1 *}$, P. G.C. CAMPBELL ${ }^{2}$, C. OLSEN ${ }^{3}$, L. MARTEL ${ }^{1}$, \\ B. PINEL-ALLOUL ${ }^{4}$, G. MÉTHOT ${ }^{4}$, A. HONTELA ${ }^{5}$
}

Reçu le 13 octobre 2003, accepté le 26 octobre $2004^{* *}$.

\section{SUMMARY}

\begin{abstract}
Metallothionein (MT), an intracellular metal binding protein, is recognized for its role in the regulation of essential and non-essential trace metals. The mechanisms of synthesis of this protein have been studied in the laboratory but much less work has been carried out in the field, particularly in lotic environments. According to the literature, metallothionein protects tissues from metal damage, and may be used as a direct measure of the biochemical state of the affected tissue. Metallothionein shows high affinity for group IB and IIB metals ions (e.g. $\mathrm{Cd}, \mathrm{Cu}, \mathrm{Zn}$ ), and consequently can sequester and detoxify metals. The use of MT as a biomarker for elevated levels of trace metals in aquatic environments has therefore been proposed as a tool in ecotoxicological studies. Changes at the biochemical level offer distinct advantages as biomarkers, since molecular alterations are normally the first detectable responses to environmental changes.
\end{abstract}

The present study focused on two objectives, the first of which was to identify which trace metals (silver "Ag", cadmium "Cd", copper "Cu", nickel "Ni", lead "Pb" and zinc "Zn") may induce MT synthesis in three sentinel species collected in two northern Quebec rivers (Colombière River, close to Val

1. Centre d'expertise en analyse environnementale du Québec. Ministère de l'Environnement. Complexe scientifique, boîte 45. 2700, rue Einstein, bureau D-2-205. Sainte-Foy, QC, Canada G1P-3W8. Téléphone : (418) 643-1301 \#338, Télécopie : (418) 528-1091.

2. Université du Québec, INRS-ETE, 490, rue de la Couronne, Québec, QC, Canada G1K $9 A 9$

3. COREM, 1180, rue de la Minéralogie, Québec, QC, G1N 1 X7.

4. Groupe de Recherche Interuniversitaire en Limnologie et en Environnement Aquatique (GRIL), Département de Sciences Biologiques, Université de Montréal, CP 6128, succursale Centre-ville, Montréal, QC Canada $\mathrm{H} 3 \mathrm{C} 3 \mathrm{~J} 7$.

5. Université du Québec à Montréal, Département des Sciences biologiques, CP 8888, Succ. Centre Ville, Montréal, OC, Canada H3C 3P8.

* Correspondance: stephane.masson@mddep.gouv.qc.ca

* Les commentaires seront reçus jusqu'au 31 mai 2006. 
d'Or, and Matagami River close to the town of Matagami) in a mining region (Abitibi, Québec). The three sentinel species were the adult bivalve Pyganodon grandis, the larval mayfly Hexagenia limbata, and the adult great northern pike Esox lucius. The second objective was to define variables that could influence the uptake of these trace metals and consequently the concentrations of MT in the sentinel species. The selection of these sentinel species was based on the following criteria: their relative abundance in the aquatic systems studied; their relative immobility; the ease of sampling; metal tolerance; metal bioaccumulation capacity; dynamics of metal accumulation; capacity to synthesize metallothionein; and availability of physiological and behavioural data.

In 2001, physico-chemical conditions (e.g.: temperature, oxygen, transparency, phosphorus, chlorophyll $a$, etc.) were monitored during two sampling campaigns at 21 stations. Trace metals were measured in water and sediment in all stations, and in tissues of the sentinel species that were collected when present at the station ( $P$. grandis - gills; $H$. limbata - whole body; E. lucius - kidney). Dialysis cells were used to collect water samples for the determination of concentrations of total dissolved metals, major anions and cations, humic and fulvic acids, and dissolved organic carbon. At each sampling site, divers collected three replicate sub-samples from two sediment cores for metal analyses. The cores were extruded in the boat and samples taken from the uppermost $0.5 \mathrm{~cm}$, i.e. from the oxidized layer.

The two rivers differ in their morphometry, their physico-chemistry, and their biology. The Allard River is larger $(200 \mathrm{~m} v s .7 \mathrm{~m})$ and deeper $(30 \mathrm{~m} v \mathrm{~s}$. $3 \mathrm{~m}$ ) than the Colombière River. The Colombière River is slightly more acid (pH: $6.2-6.7$ ) and less productive $\left(14-32 \mu \mathrm{g}\right.$ total $\left.\mathrm{P} \mathrm{L}^{-1}\right)$ than the Allard River (pH: $6.7-7.4 ; 24-45 \mu \mathrm{g}$ total $\mathbf{P ~}^{-1}$ ). However, the rivers present some similarities such as slow current conditions and limited water transparencies. The range of $\mathrm{Ag}, \mathrm{Cu}$ and $\mathrm{Ni}$ concentrations in water and sediment was greater in the Colombière River, whereas $\mathrm{Pb}$ concentrations were higher in the Allard River and Cd concentrations were similar in the two rivers. Zinc was the dominant metal in both rivers, but variations were more significant in water in the Colombière River. Although trace metal and MT concentrations were highly variable among species, stations and rivers, no consistent spatial gradients were detected in animals collected downstream from mining activities.

As was the case for the ambient environment, $Z n$ was also the dominant metal in organisms of both rivers. Zinc concentrations were similar in mayflies from the two rivers, but higher in molluscs and lower in pike collected from the Colombière River than in comparable animals from the Allard River. Following $\mathrm{Zn}, \mathrm{Cu}$ was the second metal in importance in species from the two rivers, whereas the concentrations of Ag were low in all organisms and were undetectable in pike. Cadmium was more concentrated in mayflies and molluscs from the Colombière River than in those from Allard River, and also higher in the pike collected in the contaminated zone of the Colombière River. The concentrations of $\mathrm{Ni}$ in all species were comparable between rivers, but were more elevated in mayflies than in the other sentinel species. The range of $P b$ concentrations was comparable between rivers for all species, but concentrations were lower in the pike (kidney). The concentration of MT was higher in the pike than in the two other species. Mayflies had higher MT levels in the Allard River, while MT concentrations were higher in molluses of the Colombière River. The relationships between metals and MT were very variable between rivers and species. Nevertheless, MT concentrations were most strongly related to those of accumulated $\mathrm{Cd}$ in the three sentinel species.

The relationships between MT and Cd for all three species were stronger in the Allard River than in the Colombière River. In the case of $P$. grandis, 
because the number of stations where the bivalves could be collected from the Colombière River was low $(n=4)$, no statistically significant relationship was observed between MT and Cd in the tissues, in spite of the high correlation coefficient $(r=0.98)$. For the bivalves, manganese (Mn) concentrations in the sediment were negatively correlated with accumulated $\mathrm{Cd}$ concentrations and with MT levels. In $\boldsymbol{H}$. limbata, in addition to Cd, other metals such as $\mathrm{Cu}(-)$ and $\mathrm{Zn}(+)$ also exhibited weak relationships with MT. Finally, in bivariate analyses, $\mathrm{Pb}$ concentrations in pike liver were significantly correlated with those of MT in this species, but based on multiple regression models, no variable other than $\mathrm{Cd}$ affected the level of MT in $E$. lucius.

Keywords: trace metals, cadmium, metallothionein, mayfly, bivalve mollusc, pike.

\section{RÉSUMÉ}

La métallothionéine (MT) est un ligand protéinique intracellulaire important à cause de son implication dans les processus de régulation des métaux traces essentiels et non essentiels. Bon nombre de travaux ont été conduits en laboratoire pour bien comprendre les mécanismes de synthèse de cette protéine, mais les études réalisées en milieu aquatique sont beaucoup plus rares, particulièrement dans les rivières (milieu lotique). Dans ce contexte, la présente étude avait deux objectifs principaux : (1) identifier en milieu lotique les métaux traces (argent « $\mathrm{Ag}$ », cadmium « $\mathrm{Cd}$ », cuivre « $\mathrm{Cu}$ », nickel « $\mathrm{Ni}$ », plomb « $\mathbf{P b}$ » et zinc « $\mathbf{Z n} »)$ susceptibles d'induire la synthèse de la MT chez trois espèces sentinelles, soit le mollusque bivalve Pyganodon grandis, la larve d'éphémère Hexagenia limbata et le grand brochet du nord Esox lucius ; (2) définir les variables physico-chimiques ou toxicologiques pouvant influencer la prise en charge des métaux traces et conséquemment les concentrations en MT chez le mollusque $P$. grandis et l'éphémère $H$. limbata. Deux rivières de la région de l'Abitibi ont été échantillonnées à l'été 2001 afin de récolter les espèces sentinelles à 21 stations. Les concentrations en métaux traces, ainsi qu'en métallothionéine, ont été mesurées dans les branchies des mollusques, dans le rein des brochets et dans le corps entier des éphémères. La métallothionéine a été mesurée à l'aide d'une technique de saturation au mercure. D'après nos résultats, le cadmium tissulaire semble être le meilleur prédicteur de la concentration de MT chez les trois espèces sentinelles. Cependant, l'implication d'autres métaux est également possible. Dans le cas de $P$. grandis, la concentration de manganèse (Mn) dans les sédiments pourrait influencer la prise en charge du Cd et la synthèse de MT.

Mots clés : métaux traces, cadmium, métallothionéine, éphémère, mollusque, bivalve, brochet.

\section{I - INTRODUCTION}

La pollution des rivières et des lacs par les métaux est un problème environnemental important en Amérique du Nord (NRIAGU, 1990 ; SMITH, 1987). Une fois introduits dans les écosystèmes aquatiques, les métaux tendent à s'accumuler dans les sédiments où ils deviennent une menace potentielle pour les organismes benthiques. Dans de tels environnements, les animaux benthiques accumulent les métaux soit de l'eau avec laquelle ils sont continuellement en contact, soit via la nourriture qu'ils ingèrent (HARE, 1992). 
La synthèse de ligands protéiques spécifiques aux métaux est un de moyens de protection des organismes aux effets de la contamination métallique (ROESIJADI, 1980 ; VIARENGO et al., 1999). Ces ligands protéiniques conjuguent, lient et détoxiquent les métaux (CAMPBELL et COUILLARD, 2004). La métallothionéine (MT) est reconnue comme un ligand protéinique qui joue un rôle important dans le contrôle des métaux essentiels comme le cuivre et le zinc, ainsi que des métaux non essentiels comme l'argent, le cadmium et le mercure (KAGl et KOJIMA, 1987; ROESIJADI, 1996). La fonction majeure de la MT est associée à la régulation homéostatique des métaux intracellulaires (VIARENGO, 1989).

En milieu lacustre, plusieurs auteurs ont observé des relations significatives entre les concentrations de l'ion libre du Cd et celles de la MT (ex. : COUILLARD et al., 1993 ; CROTEAU et al. 2002 ; PERCEVAL et al., 2002) ainsi qu'entre certains métaux $(\mathrm{Cd}, \mathrm{Cu}, \mathrm{Zn})$ dans des organismes et la MT (ex. : diptères : CROTEAU et al., 2002 ; éphémères : COUILLARD, 1997; mollusques: CoUILLARD et al., 1993; PERCEVAL et al., 2002 ; poissons : LAFLAMME et al., 2000 ; OLSVIK et al., 2000 et 2001). Par ailleurs, très peu d'études ont porté sur les relations métaux-MT en rivières (BAUDRIMONT et al., 1999 ; FARAG et al., 1995 et 2003). Le régime hydrologique et les caractéristiques physico-chimiques en rivières peuvent varier fréquemment selon les précipitations. Les rivières sont donc des milieux dynamiques, où les interactions entre la contamination métallique et les concentrations en MT chez les organismes peuvent s'avérer très différentes de celles observées en milieu lacustre.

Le principal objectif de ce travail était de déterminer s'il existait, en milieu lotique, des liens entre la MT et six métaux $(\mathrm{Ag}, \mathrm{Cd}, \mathrm{Cu}, \mathrm{Ni}, \mathrm{Pb}, \mathrm{Zn})$ mesurés chez trois espèces sentinelles, soit le mollusque bivalve Pyganodon grandis, la larve d'éphémère Hexagenia limbata, ainsi que le grand brochet du nord Esox lucius. Comme second objectif, nous voulions déterminer si les métaux qui induisent la synthèse de la MT diffèrent entre les espèces. Finalement, nous voulions identifier les facteurs physico-chimiques et/ou toxicologiques (concentrations des métaux) dans le milieu ou chez les organismes qui peuvent améliorer la performance des modèles développés pour prédire les concentrations en MT. Nous émettons l'hypothèse que le Cd est le métal influençant le plus la concentration de MT chez les trois espèces sentinelles.

\section{2 - MATÉRIEL ET MÉTHODES}

\section{$2.1 \quad$ Milieu d'étude}

L'échantillonnage a été réalisé au nord-ouest du Québec, Canada, dans deux rivières situées dans la région minière de l'Abitibi, soit la rivière Colombière située près de $\mathrm{Val}$ d'Or et la rivière Allard localisée près de Matagami (figure 1). Vingt-et-une stations distribuées le long des deux rivières ont été échantillonnées au cours de l'été 2001. Douze stations ont été établies dans la rivière Allard (AL1 à AL12), tandis que neuf stations ont été positionnées dans la rivière Colombière (CO1 à CO9). Les stations étaient séparées d'environ deux kilomètres afin de minimiser l'effet d'autocorrélation spatiale, de diminuer 
les chances que les mêmes populations de poissons fréquentent deux zones adjacentes, ainsi que pour maximiser les différences au niveau des caractéristiques physico-chimiques et des concentrations ambiantes des métaux ciblés pour cette étude. Les stations furent choisies sur la base de la présence d'au moins une espèce sentinelle.

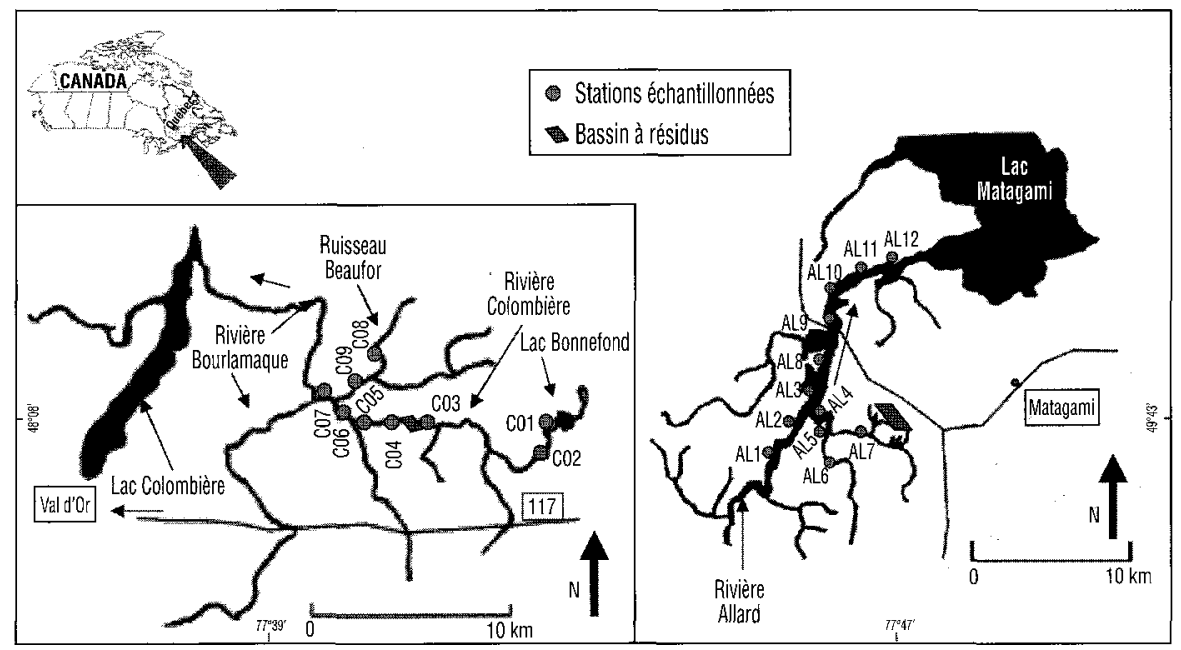

Figure 1 Position des stations échantillonnées dans les rivières Allard et Colombière, Québec, Canada.

Sampling stations in the Allard and Colombière Rivers, Québec, Canada.

\subsection{Récolte d'eau et de sédiments pour l'analyse des métaux}

Au cours de deux campagnes d'échantillonnage (début-juillet et mi-août) des dialyseurs de $250 \mathrm{ml}$ de volume, munis de membranes filtrantes d'une porosité de $0,45 \mu \mathrm{m}$, ont été placés pour une période de deux semaines dans la zone oxyque $(0,5 \mathrm{~m}$ du fond) à chacune des stations afin de déterminer les concentrations en métaux totaux dissous. Par la suite, les échantillons d'eau des dialyseurs ont été conservés au froid et expédiés au laboratoire central pour analyse dans les $24 \mathrm{~h}$ suivant l'envoi. Par la suite, les éléments traces étaient mesurés soit par spectroscopie d'émission atomique couplé à un plasma (ICP-AES), par spectroscopie de masse d'émission atomique couplé à un plasma (ICP-MS) et/ou par spectrophotométrie d'absorption atomique avec four au graphite (SAA-FG).

À chaque station et à une seule campagne, des carottes de sédiments étaient récoltées à trois endroits en plongée sous-marine dans le but de déterminer la concentration totale des métaux sédimentaires dans la couche superficielle oxyque $(0,5 \mathrm{~cm})$. Les échantillons ont été conservés à $-20^{\circ} \mathrm{C}$ jusqu'au moment de l'analyse qui fut réalisée à l'automne. Les détails des procédures d'extraction et d'analyse en laboratoire sont donnés dans COUILLARD et al. (1993). 


\subsection{Récolte des espèces sentinelles et dosage des métaux et de la MT dans les organismes}

Des spécimens adultes et d'âge similaire de $P$. grandis $(5-6$ ans ; $7-9 \mathrm{~cm}$; $n=9$ lorsque possible) ont été récoltés en plongée sous-marine avant le début de la période de gravidité (fin juin - début juillet) et gardés vivants dans des glacières remplies d'eau de la rivière jusqu'au moment des dissections, i.e. à la fin de l'après-midi suivant l'échantillonnage. Les mollusques ont été trouvés à quatre stations dans la rivière Colombière ( $\mathrm{CO} 1, \mathrm{CO} 2, \mathrm{CO} 8$ et $\mathrm{CO} 9$ ), tandis qu'ils étaient présents à huit stations dans la rivière Allard (AL1 à AL3 et AL8 à $\mathrm{AL12}$ ). Les branchies de droite de trois mollusques étaient réunies pour le dosage de la métallothionéine, tandis que celles de gauche étaient conservées pour le dosage des métaux. Cette procédure a été réalisée afin d'obtenir trois échantillons composites de trois mollusques par site lorsque possible.

Simultanément, les larves de $H$. limbata $(1,5$ à $2 \mathrm{~mm} ; n=60)$ étaient récoltées en tamisant la surface des sédiments à l'aide d'un filet benthique manipulé par des plongeurs. Un temps d'attente de $24 \mathrm{~h}$ était nécessaire à la dépuration des éphémères avant de regrouper dix individus pour obtenir trois échantillons composites par site pour les dosages des métaux et trois autres échantillons pour ceux de la MT. Les éphémères ont été trouvées à toutes les stations de la rivière Allard à l'exception de la station $A L 7$, tandis qu'elles étaient absentes aux stations $\mathrm{CO} 2$ et $\mathrm{CO} 5$ de la rivière Colombière.

Finalement, le brochet $E$. lucius $(50-90 \mathrm{~cm} ; n=8)$ a été capturé dans deux zones (référence et contaminée) dans les deux rivières. Les brochets ont été capturés au lancer léger ou à l'aide de filets placés dans la colonne d'eau pendant de courtes périodes $(<2 \mathrm{~h})$. Dans la rivière Colombière, la capture des brochets a été réalisée entre $\mathrm{CO} 1$ et $\mathrm{CO} 2$ pour la zone de référence et entre $\mathrm{CO} 3$ et $\mathrm{CO} 7$ pour la zone contaminée (figure 1). La zone de référence de la rivière Allard était positionnée en amont de la station $\mathrm{AL2}$, tandis que la zone contaminée était localisée entre AL3 et AL5. Au laboratoire, trois sous-échantillons de rein étaient prélevés sur chaque poisson pour les dosages respectifs des métaux et de la MT.

Des échantillons homogénéisés (100 à $700 \mu$ g poids sec) de l'organisme entier $(H$. limbata) ou des organes spécifiques (branchies pour les mollusques et reins pour le brochet) ont servi à la détermination des métaux ( $\mathrm{Ag}, \mathrm{Cd}, \mathrm{Cu}$, $\mathrm{Ni}, \mathrm{Pb}, \mathrm{Zn}$ ). Au préalable, ces broyats étaient séchés au lyophilisateur pour un minimum de $5 \mathrm{j}$. De l'acide nitrique ultrapur (2 à $20 \mathrm{ml}$ selon la quantité de tissu) était ajouté pour effectuer une prédigestion sur une période de $24 \mathrm{~h}$ à la température de la pièce. Par la suite, les sous-échantillons de ces digestats étaient transférés dans des bombes de digestion en Téflon afin de compléter la digestion dans un autoclave pour une période de $3 \mathrm{~h}$ à une pression de $400 \mathrm{~mm} \mathrm{Hg}$. Les digestats refroidis étaient dilués avec de l'eau déionisée ultrapure pour obtenir un volume final de $10 \mathrm{ml}$. La concentration des métaux a été déterminée à l'aide d'un ICP-AES. Des blancs analytiques et du matériel de référence certifié (TORT-2 : hépatopancréas de homard, Marine Analytical Chemistry Standards Program, National Research Council of Canada, Ottawa, ON) ont été analysés au cours de la procédure analytique des échantillons des trois espèces. L'analyse de ces échantillons indiquait une contamination négligeable pour les blancs et une récupération totale pour le cadmium (110\%) et près des valeurs certifiées pour l'ensemble des autres métaux (91 à $109 \%$ ). 
Pour les analyses de la MT, un sous-échantillon de broyat frais pour chaque échantillon d'organe pour les trois espèces était centrifugé à $30,000 \times \mathrm{g}$ pendant une période de 30 min à une température de $4{ }^{\circ} \mathrm{C}$. Le surnageant était analysé pour la MT à l'aide d'une technique de saturation au mercure dont la méthode est décrite plus en détail dans CoUILLARD et al. (1993). Comme contrôle de qualité, le pourcentage de récupération d'un standard de MT (foie de lapin, Sigma Chemical Co.) a été déterminé à chaque essai analytique. La récupération moyenne de MT pour dix essais était de 106,6 $\pm 1,4 \%$.

\subsection{Analyses statistiques}

Des corrélations de Pearson ont été établies entre les six métaux ( $\mathrm{Ag}, \mathrm{Cd}$, $\mathrm{Cu}, \mathrm{Ni}, \mathrm{Pb}, \mathrm{Zn}$ ) et la MT mesurés chez les trois espèces sentinelles pour chacune des rivières séparément et par la suite en combinant les deux rivières. Les niveaux de signification des corrélations ont tous été estimés à l'aide de tests par permutations (LEGENDRE et LEGENDRE, 1998). Une correction de Bonferroni a été appliquée à l'ensemble des tests. Des analyses de régression multiple avec l'option d'une sélection progressive "forward selection " ont été réalisées afin d'identifier quels étaient les facteurs, parmi les variables physico-chimiques du milieu et celles liées à la contamination du milieu ou des organismes, qui permettaient de mieux prédire la MT chez les trois espèces sentinelles. La concentration de MT dans le corps complet $(H$. limbata) ou dans les organes des deux autres espèces (branchies pour mollusques et reins pour le brochet) était la variable dépendante, tandis que les variables indépendantes étaient la concentration des six métaux dans les mêmes organes, la physicochimie des eaux, ainsi que la concentration des métaux dans l'eau et les sédiments. La procédure de sélection progressive était interrompue lorsque l'effet de la variable additionnelle choisie avait un seuil de signification $>0,05$. Une transformation logarithmique des données $\left(\log _{10}\right)$ a été appliquée pour linéariser les relations entre les variables. Des tests de Kolmogorov-Smirnov ont été utilisés pour vérifier que les résidus du modèle de régression linéaire étaient normalement distribués et l'homoscédasticité des résidus a été vérifiée visuellement par l'examen des relations entre les résidus et les valeurs prédites. Les analyses de régressions linéaires simples et multiples ont été réalisées à l'aide du logiciel SYSTAT version 8.

\section{3 - RÉSULTATS}

\subsection{Concentrations des métaux traces dans l'environnement}

Les étendues de concentrations des métaux mesurées dans l'eau et les sédiments sont présentées au tableau 1. Selon le métal, il existe des niveaux de contamination différents entre les deux rivières et une variabilité spatiale de contamination au sein de chacune des rivières. La rivière Colombière présentait des concentrations plus élevées en $\mathrm{Ag}$ et $\mathrm{Cu}$, à la fois dans l'eau et les sédiments. De plus, l'étendue de variation des concentrations y était beaucoup plus importante selon le rapport de concentration Max/Min (tableau 1). Les 
valeurs de cadmium dans les sédiments étaient comparables dans les deux rivières. Le plomb dans les sédiments était plus concentré dans la rivière Allard et variait plus entre les stations que dans la rivière Colombière. Le zinc était le métal dominant dans les rivières Colombière et Allard, avec des concentrations maximales de l'ordre de $800 \mathrm{nM}$ et $900 \mathrm{nM}$ dans l'eau et de $12000 \mathrm{nmol} \cdot \mathrm{g}^{-1}$ et $15000 \mathrm{nmol} \cdot \mathrm{g}^{-1}$ dans les sédiments. L'étendue de variation était plus importante dans la rivière Colombière, autant pour la colonne d'eau que pour les sédiments. Dans la rivière Allard, sauf pour le $\mathrm{Cu}$ et le $\mathrm{Ni}$ dans l'eau, les stations les plus concentrées en métaux dans l'eau et les sédiments se situaient en aval du bras drainant le bassin à résidu minier (AL4, AL5 et AL7). Dans la rivière Colombière, la distribution des concentrations maximales était plus variable selon les métaux. La concentration des métaux aurait dû décroître de la station $\mathrm{CO} 4$ vers $\mathrm{CO} 7$ suivant l'éloignement du bras drainant le bassin à résidu minier. Cependant, les concentrations en $\mathrm{Ag}$ et en $\mathrm{Cu}$ étaient plus élevées en amont de ce bras (CO1 à $\mathrm{CO} 3$ ).

Tableau 1 Étendue des concentrations de métaux mesurées dans l'eau (total dissous) et des sédiments aux stations des deux rivières.

Table 1 Range of metal concentrations measured in the water and sediment at stations in our study rivers.

\begin{tabular}{|c|c|c|c|c|c|}
\hline & \multicolumn{2}{|c|}{ Eau (nM) } & \multicolumn{2}{|c|}{ Sédiment $\left(\mathrm{nmol} \cdot \mathrm{g}^{-1}\right)$} \\
\hline & & Allard & Colombière & Allard & Colombière \\
\hline \multicolumn{6}{|c|}{ Métaux } \\
\hline & $\begin{array}{l}\text { Étendue } \\
\text { Max/Min }\end{array}$ & $\begin{array}{c}\left\langle 9,3\left(^{*}\right)-26,4(\text { AL } 7)\right. \\
2,8\end{array}$ & $\begin{array}{c}12(\mathrm{CO} 7)-59(\mathrm{CO}) \\
4,9\end{array}$ & $\begin{array}{c}1,6 \text { (AL1) - 14,2 (AL5) } \\
8,9\end{array}$ & $\begin{array}{c}<1(\mathrm{CO})-83(\mathrm{CO}) \\
83\end{array}$ \\
\hline Cd & $\begin{array}{l}\text { Étendue } \\
\text { Max/Min }\end{array}$ & $<8,9\left(^{\star}\right)$ & $\left.<8,94^{*}\right)$ & $\begin{array}{c}2,9(\mathrm{AL} 2)-15,3(\mathrm{AL} 4) \\
5,3\end{array}$ & $\begin{array}{c}<8,9(\mathrm{CO})-17,2(\mathrm{CO} 4) \\
1,9\end{array}$ \\
\hline & $\begin{array}{l}\text { Étendue } \\
\text { Max/Min }\end{array}$ & $\begin{array}{c}0,04(\mathrm{AL} 6)-0,7(\mathrm{AL} 5) \\
18,5\end{array}$ & $\begin{array}{c}0,03(\mathrm{CO})-0,5(\mathrm{CO} 6) \\
17,7\end{array}$ & - & - \\
\hline & $\begin{array}{l}\text { Étendue } \\
\text { Min }\end{array}$ & $\begin{array}{c}<16\left(^{*}\right)-74(\mathrm{AL} 8) \\
4,7\end{array}$ & $\begin{array}{c}24(\mathrm{CO})-270(\mathrm{CO}) \\
11,1\end{array}$ & $\begin{array}{c}278 \text { (AL1) }-2329 \text { (AL7) } \\
8,4\end{array}$ & $\begin{array}{c}220(\mathrm{CO} 2) \cdot 3288(\mathrm{CO}) \\
14,9\end{array}$ \\
\hline & $\begin{array}{l}\text { Étendue } \\
\text { Max/Min }\end{array}$ & $\begin{array}{c}<17\left(^{\star}\right)-25(A L 6) \\
1,5\end{array}$ & $\begin{array}{c}<17\left(^{\star}\right)-52(\mathrm{CO5}) \\
3,1\end{array}$ & $\begin{array}{c}642 \text { (AL5) - } 935 \text { (AL7) } \\
1,5\end{array}$ & $\begin{array}{c}504 \text { (CO1) - } 1533(\mathrm{CO} 9) \\
3,0\end{array}$ \\
\hline & $\begin{array}{l}\text { Étendue } \\
\text { Max/Min }\end{array}$ & $<4,8\left(^{*}\right)$ & $<4,8\left(^{*}\right)$ & $\begin{array}{c}102(\mathrm{AL} 1)-270(\mathrm{AL} 5) \\
2,6\end{array}$ & $\begin{array}{c}70(\mathrm{CO} 2)-119(\mathrm{CO} 5) \\
1,7\end{array}$ \\
\hline & $\begin{array}{l}\text { Étendue } \\
\text { Min }\end{array}$ & $\begin{array}{c}42 \text { (AL10) - } 910(A L 7) \\
21,9\end{array}$ & $\begin{array}{c}<15(\mathrm{CO} 2)-824(\mathrm{CO} 6) \\
53,9\end{array}$ & $\begin{array}{c}1537 \text { (AL1) - } 15170 \text { (AL7) } \\
9,9\end{array}$ & $\begin{array}{c}999 \text { (CO8) - } 12402(\mathrm{CO} 4) \\
12,4\end{array}$ \\
\hline & $\begin{array}{l}\text { Étendue } \\
\text { Min }\end{array}$ & $\begin{array}{c}32 \text { (AL2) - } 494 \text { (AL7) } \\
15,7\end{array}$ & $\begin{array}{c}30(\mathrm{CO})-618(\mathrm{CO} 6) \\
20,4\end{array}$ & - & - \\
\hline
\end{tabular}

* : concentration sous la limite de détection pour la majorité des stations.

Lorsque les concentrations minimales sont à la limite de détection, le ratio Max/Min est calculé à partir de la valeur de la limite de détection (LDD).

LDD pour le Cd : 0,5 ug- L-1 pour l'ICP-AES ; 0,1 ug. L-1 pour le SAA-FG et 0,01 ug. L-1 pour l'ICP-MS.

LDD pour le $Z n: 1$ ug. $\mathrm{L}^{-1}$ pour l'ICP-AES et de $0,01 \mathrm{ug} \cdot \mathrm{L}^{-1}$ pour les deux autres appareils.

Lorsque les concentrations en Cd et en $\mathrm{Zn}$ sont $10 x$ plus élevées que la limite de détection, l'erreur analytique est similaire pour l'ensemble des appareils et se situe à entre 2 et $5 \%$. 


\subsection{Concentrations des métaux et de MT dans les espèces sentinelles}

Les concentrations des six métaux et de la MT mesurées dans les trois espèces sentinelles sont présentées au tableau 2. Parmi les six métaux, le Zn était le métal en plus grande concentration chez les trois espèces sentinelles dans les deux rivières. La concentration des autres métaux variait selon l'espèce, la station et la rivière. Même si les stations AL1-AL2 et CO1-CO2 étaient considérées à l'origine comme des sites de référence, aucun gradient de concentration polymétallique tissulaire amont $\rightarrow$ aval n'était détectable dans les deux rivières.

Dans le corps entier de l'éphémère $H$. limbata, l'étendue de concentrations de $\mathrm{Zn}$ était comparable entre les deux rivières et variait respectivement de 220 à $383 \mu \mathrm{g} \cdot \mathrm{g}-1$ et de 190 à $455 \mu \mathrm{g} \cdot \mathrm{g}^{-1}$ dans les rivières Colombière et Allard (tableau 2). Le Cu (16,8 à 72,4 $\left.\mu \mathrm{g} \cdot \mathrm{g}^{-1}\right)$ était le second métal en importance dans les individus de la rivière Colombière, tandis que ses concentrations étaient plus faibles dans les individus de la rivière Allard $\left(14,5\right.$ à $\left.34,8 \mu \mathrm{g} \cdot \mathrm{g}^{-1}\right)$. À l'inverse, le $\mathrm{Cd}$ était plus concentré dans les individus de la rivière Allard $(2,5$ à $16,8 \mu \mathrm{g} \cdot \mathrm{g}^{-1}$ ) et moins concentré chez les éphémères de la rivière Colombière $\left(0,9\right.$ à $\left.5,0 \mu \mathrm{g} \cdot \mathrm{g}^{-1}\right)$. Les concentrations d'Ag étaient faibles dans les organismes des deux rivières, variant de 0,03 à $0,45 \mu \mathrm{g} \cdot \mathrm{g}^{-1}$ dans la rivière Colombière et de 0,08 et $0,26 \mu \mathrm{g} \cdot \mathrm{g}^{-1}$ dans la rivière Allard. Les concentrations de $\mathrm{Ni}$ et de $\mathrm{Pb}$ étaient similaires dans les deux rivières $\left(\leq 10,4 \mu \mathrm{g} \cdot \mathrm{g}^{-1}\right)$. La concentration totale de MT était nettement plus élevée dans les éphémères de la rivière Allard (50 à $173 \mathrm{nmol}$ sites. $\mathrm{g}^{-1}$ poids $\mathrm{sec}$ ) que dans la rivière Colombière (39 à $58 \mathrm{nmol}$ sites. $\mathrm{g}^{-1}$ poids sec).

Dans les branchies du mollusque $P$. grandis, les concentrations en $\mathrm{Zn}$ étaient plus importantes dans la rivière Allard (502 à $920 \mu \mathrm{g} \cdot \mathrm{g}^{-1}$ ) que dans la rivière Colombière (390 à $484 \mu \mathrm{g} \cdot \mathrm{g}^{-1}$ ) (tableau 2). Parmi les autres métaux, le $\mathrm{Cu}$ était le second plus important dans les branchies. II était notamment plus concentré aux stations CO8 et CO9 dans la rivière Colombière $(29,1$ et $\left.21,8 \mu \mathrm{g} \cdot \mathrm{g}^{-1}\right)$ et aux stations AL3 et AL8 dans la rivière Allard $\left(19,2\right.$ et $\left.15,5 \mu \mathrm{g} \cdot \mathrm{g}^{-1}\right)$. Le Cd était aussi plus concentré chez les individus de la rivière Colombière $(3,2$ à $8,0 \mu \mathrm{g} \cdot \mathrm{g}^{-1}$ ) que chez ceux de la rivière Allard (2 à $12 \mu \mathrm{g} \cdot \mathrm{g}^{-1}$ ). La concentration du Ni était très faible dans les branchies des mollusques $\left(\leq 2 \mu \mathrm{g} \cdot \mathrm{g}^{-1}\right)$ comparativement aux éphémères $\left(\leq 10 \mu \mathrm{g} \cdot \mathrm{g}^{-1}\right)$, tandis que la concentration en $\mathrm{Ag}$ y était plus importante (max : 3,6 vs. $0,3 \mu \mathrm{g} \cdot \mathrm{g}^{-1}$ respectivement). La concentration de $\mathrm{Pb}$ était plus élevée dans les branchies des mollusques de la rivière Colombière $\left(4,9\right.$ à $\left.7,9 \mu \mathrm{g} \cdot \mathrm{g}^{-1}\right)$ que dans la rivière Allard $\left(0,7\right.$ à $\left.4,9 \mu \mathrm{g} \cdot \mathrm{g}^{-1}\right)$. À l'inverse des larves d'éphémères, la concentration totale de MT était plus grande dans les mollusques de la rivière Colombière $\left(37,3\right.$ à $71,9 \mathrm{nmol}$ sites $\cdot \mathrm{g}^{-1}$ poids sec) que dans la rivière Allard ( 3,2 à $45,8 \mathrm{nmol}$ sites. $\mathrm{g}^{-1}$ poids sec). Par contre, le rapport Max/Min de la MT était plus grand pour la rivière Allard que dans la rivière Colombière (14 vs. 2) ; en effet, les concentrations de MT étaient plutôt élevées chez les mollusques des stations dites de référence de la rivière Colombière, soit $\mathrm{CO} 1$ et $\mathrm{CO} 2$. 
Tableau 2 Concentrations moyennes des métaux ( $\mu \mathrm{g} \cdot \mathrm{g}^{-1}$ poids secs) et de la métallothionéine (MT : $\mathrm{nmol}$ sites $\cdot \mathrm{g}^{-1}$ poids secs) dans les organes de trois espèces sentinelles prélevées dans les rivières Colombière et Allard (espaces blancs : absence de l'espèce ; n.d. : métal sous la limite de détection).

Table 2 Mean metal ( $\mu \mathrm{g} \cdot \mathrm{g}^{-1}$ dry weight) and metallothionein (MT: nmol sites. $\mathrm{g}^{-1}$ dry weight) concentrations in three sentinel species collected in the Colombière and Allard rivers (blank space: species not collected; $n . d$.: below detection limit).

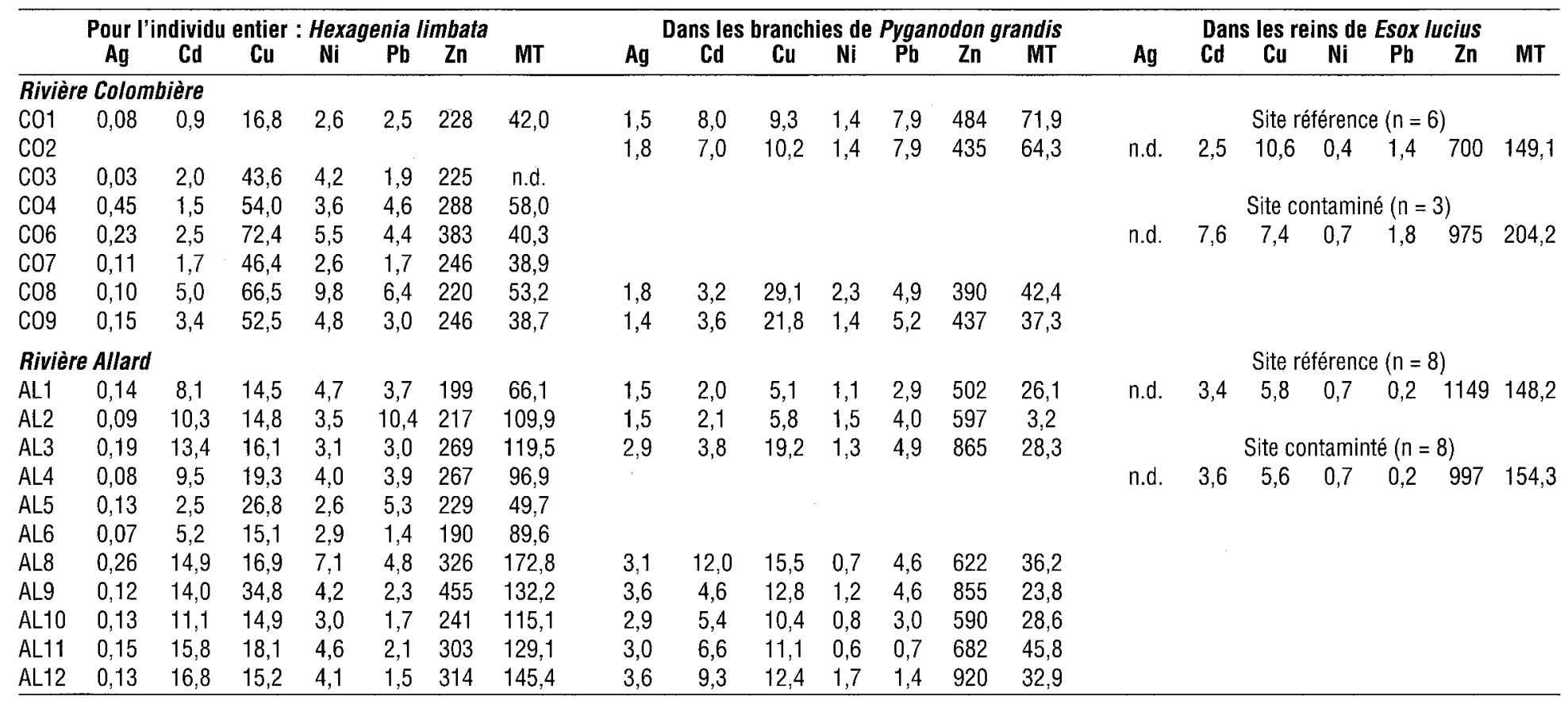


Chez les brochets de la rivière Colombière, à l'exception du $\mathrm{Cu}$, toutes les concentrations moyennes de métaux et de MT étaient plus basses dans la zone de référence que dans la zone contaminée (tableau 2). La concentration en $\mathrm{Cu}$ était légèrement plus élevée dans le rein des brochets du site de référence $\left(10,6\right.$ vs. $\left.7,4 \mu \mathrm{g} \cdot \mathrm{g}^{-1}\right)$ et ces concentrations étaient plus grandes que celles observées dans les reins des brochets capturés dans la rivière Allard $\left(5,6-5,8 \mu \mathrm{g} \cdot \mathrm{g}^{-1}\right)$. Par contre dans la rivière Allard, les concentrations moyennes de $\mathrm{Cd}, \mathrm{Cu}, \mathrm{Ni}, \mathrm{Pb}, \mathrm{Zn}$ et MT chez le brochet étaient comparables entre les sites de référence et contaminés (tableau 2). L'argent n'a pas été détecté dans les reins des brochets des deux rivières. Les concentrations de MT étaient plus élevées dans le rein des brochets capturés dans le site contaminé de la rivière Colombière (204 $\mathrm{nmol}$ sites. $\mathrm{g}^{-1}$ poids $\mathrm{sec}$ ), tandis que les concentrations aux trois autres sites étaient comparables (148 à $154 \mathrm{nmol}$ sites. $\mathrm{g}^{-1}$ poids sec). Le faible nombre de poissons ne permettait pas de tester les différences observées entre les sites d'une même rivière.

\subsection{Relations entre la MT et les six métaux dans les trois espèces sentinelles}

Nous avons établi dans un premier temps des corrélations entre les métaux tissulaires et la MT des trois espèces sentinelles pour chacune des deux rivières de façon indépendante et par la suite en combinant les deux rivières (tableau 3). Le Cd semble être le métal le plus fortement corrélé avec la concentration de MT chez les trois espèces sentinelles. Chez l'éphémère $H$. limbata, la relation entre ces variables est significative uniquement dans la rivière Allard ( $r=0,89 ; P \leq 0,001)$ et le niveau de corrélation devient plus élevé en combinant les deux rivières $(r=0,94 ; P \leq 0,001)$. La relation entre la MT et le $\mathrm{Cd}$ chez $P$. grandis est quelque peu différente puisque la corrélation est plus importante dans la rivière Colombière $(r=0,98 ; P>0,05)$ mais non significative en raison du faible nombre d'observations $(n=4)$. La relation est significative dans la rivière Allard $(r=0,62 ; P \leq 0,05)$ et demeure significative en combinant les deux rivières $(r=0,58 ; P=0,046)$. Le brochet est la seule espèce présentant des relations significatives entre la MT et le $\mathrm{Cd}$ dans chacune des rivières ( $r>0,51 ; P \leq 0,05$ ), et cette relation demeure significative en combinant les deux rivières $(r=0,73 ; P \leq 0,001)$.

Les éphémères de la rivière Colombière ne présentent aucune relation significative entre la MT et les cinq autres métaux. Par contre, des relations significatives $(P \leq 0,05)$ sont observées avec l'Ag $(0,54)$, le $\mathrm{Ni}(0,63)$ et le $\mathrm{Zn}(0,64)$ dans la rivière Allard (tableau 3 ). En combinant les deux rivières, ces relations ne sont plus significatives, tandis qu'on observe une relation négative très hautement significative entre le Cu et la MT ( $r=-0,61 ; P \leq 0,001)$.

Outre le Cd, aucune relation significative n'est observée entre la MT et les concentrations des autres métaux chez $P$. grandis dans les deux rivières. Le faible nombre d'observations dans la rivière Colombière influence grandement le niveau de signification puisque trois métaux présentent des corrélations supérieures à 0,9 mais non significatives, soit le $\mathrm{Cd}(0,98)$, le $\mathrm{Cu}(-0,91)$ et le $\mathrm{Pb}(0,96)$. En combinant les deux rivières, la relation entre la $\mathrm{MT}$ et le $\mathrm{Pb}$ devient significative mais le coefficient de corrélation est beaucoup plus faible $(r=0,53)$ que la relation observée dans la rivière Colombière $(r=0,96)$ (tableau 3). La relation entre les métaux et la MT chez le brochet $E$. lucius est 
également significative pour le $\mathrm{Pb}$ dans les rivières Allard et Colombière et devient très hautement significative en combinant les deux rivières bien que le coefficient de corrélation soit plus bas que pour la rivière Colombière seule (tableau 3).

Tableau 3 Matrice de corrélations entre la concentration de métallothionéine (MT) et les concentrations de métaux mesurées chez les trois espèces sentinelles pour chacune des rivières et pour les deux rivières combinées. Les corrélations significatives à un niveau de $P<0,05$ sont soulignées une fois, celles à un niveau de $P \leq 0,01$ sont doublement soulignées, et celles à un niveau de $P \leq 0,001$ sont doublement soulignées et en caractères gras. Les niveaux de signification ont été obtenus à l'aide de tests de permutations (9 999 permutations).

Table 3 Correlation matrix between metallothionein (MT) and metal concentrations measured in three sentinel species for each river and for the two rivers combined. Significant relationships at $P<0.05$ are underlined once, those at $P \leq 0.01$ are underlined twice and those at $P \leq 0.001$ are underlined twice and are in bold text. Significance levels were obtained by permutation tests (9999 permutations).

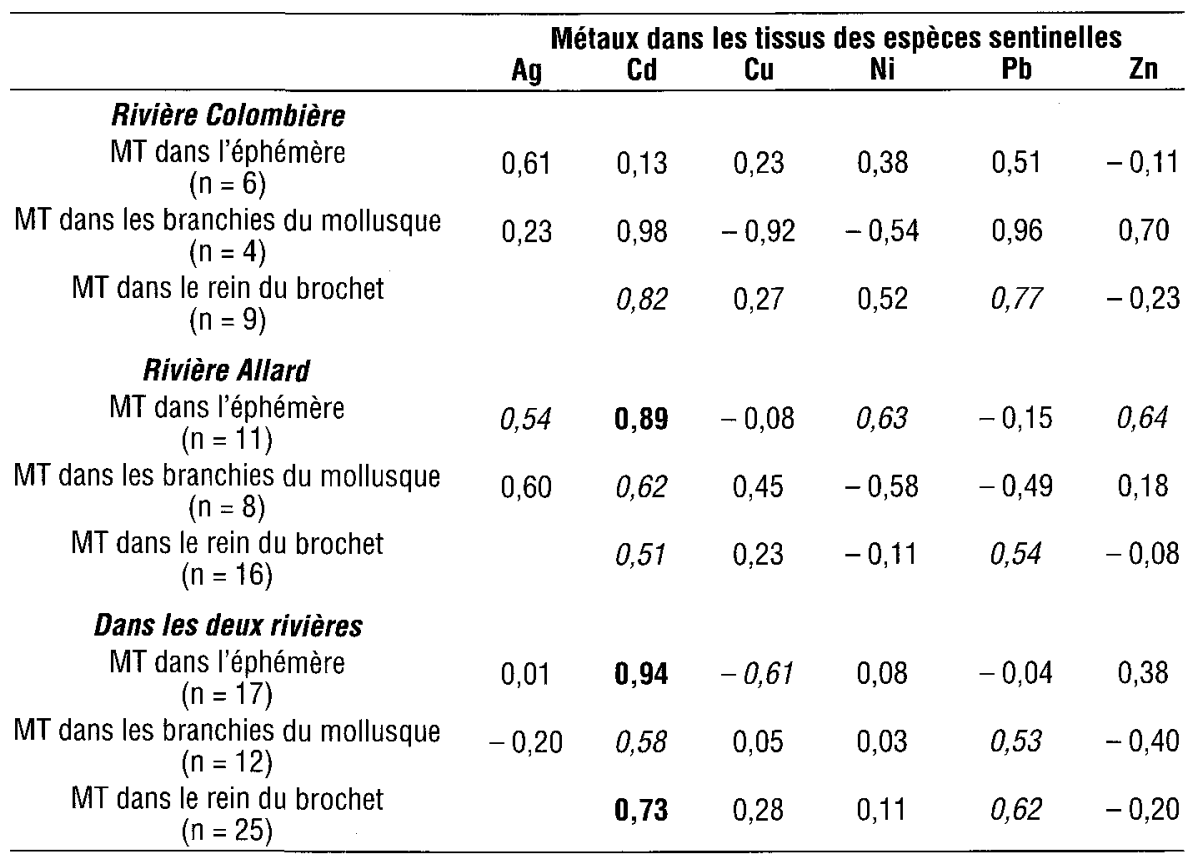

\subsection{Modèles de régression multiple prédisant la [MT] pour les trois espèces sentinelles}

Les modèles de régression multiple qui tiennent compte des données des deux rivières suggèrent que le $\mathrm{Cd}$ bioaccumulé est le principal métal influençant le niveau de MT chez les trois espèces sentinelles (tableau 4). Le Cd explique positivement plus de $80 \%$ de la MT chez $\mathrm{H}$. limbata, tandis que près 
de $9 \%$ de la variance s'explique par un effet négatif du $\mathrm{Cu}(4 \%)$ et un effet positif du Zn (5\%). Chez $P$. grandis, la concentration en Cd explique plus de $34 \%$ de la concentration MT, tandis que $27 \%$ de plus est expliqué par un effet négatif de la concentration de $\mathrm{Mn}$ dans les sédiments. Finalement, aucun autre métal à l'exception du Cd n'influence le niveau de MT chez le brochet E. lucius. Dans ce dernier modèle, il n'était pas possible de déterminer l'influence de la physico-chimie étant donné que les poissons n'avaient pas été pêchés par station mais plutôt par zone.

Tableau $4 \quad$ Modèles de régression multiple pour prédire la concentration de la métallothionéine (MT) dans les tissus des éphémères $(H L)$, dans les branchies des mollusques (PG), ainsi que dans les reins du grand brochet du nord (EL) en fonction des concentrations de métaux dans les organes des espèces, la physico-chimie des eaux et les concentrations de métaux dans les sédiments aux stations des rivières Allard et Colombière.

Table $4 \quad$ Multiple regression models to predict metallothionein (MT) concentrations in mayflies, in the gills of molluscs (PG), and in the kidney in the northern pike (EL), using metal concentrations in each species, the physico-chemistry of the Allard and Colombière rivers, and metal concentrations in the sediments.

\begin{tabular}{|c|c|c|c|c|c|}
\hline Modèle & & $\mathrm{R}^{2}$ & SE & $t$ & $P(t)$ \\
\hline Log MTHL & $\begin{array}{c}0,67 \\
+0,34 \log \mathrm{CdHL} \\
-0,35 \log \mathrm{CuHL} \\
+0,60 \log \mathrm{ZnHL}\end{array}$ & $\begin{array}{l}0,81 \\
0,85 \\
\mathbf{0 , 8 9}\end{array}$ & $\begin{array}{l}0,52 \\
0,07 \\
0,11 \\
0,25\end{array}$ & $\begin{array}{c}1,30 \\
4,84 \\
-3,21 \\
2,41\end{array}$ & $\begin{array}{c}0,218 \\
<0,001 \\
0,007 \\
0,032\end{array}$ \\
\hline Log MTPG & $\begin{array}{c}2,44 \\
+0,85 \mathrm{Log} \mathrm{CdPg} \\
-0,48 \log \mathrm{MnS}\end{array}$ & $\begin{array}{l}0,34 \\
\mathbf{0 , 6 1}\end{array}$ & $\begin{array}{l}0,65 \\
0,29 \\
0,20\end{array}$ & $\begin{array}{c}3,75 \\
2,92 \\
-2,47\end{array}$ & $\begin{array}{l}0,005 \\
0,017 \\
0,036\end{array}$ \\
\hline Log MTEL & $\begin{array}{c}1,89 \\
+0,56 \text { Log CdEL }\end{array}$ & 0,33 & $\begin{array}{l}0,09 \\
0,17\end{array}$ & $\begin{array}{c}20,05 \\
3,34\end{array}$ & $\begin{array}{c}<0,001 \\
0,003\end{array}$ \\
\hline
\end{tabular}

\section{4 - DISCUSSION ET CONCLUSIONS}

\subsection{Lien entre la MT et l'ensemble des métaux}

Le principal objectif de ce travail était de déterminer s'il existait, en milieu lotique, des liens entre la MT et six métaux ( $\mathrm{Ag}, \mathrm{Cd}, \mathrm{Cu}, \mathrm{Ni}, \mathrm{Pb}, \mathrm{Zn}$ ) mesurés chez trois espèces sentinelles. Selon MCINTOSH (1991), I'identification des effets des métaux traces sur la faune aquatique se complique par le fait que d'une part les organismes se retrouvent dans des milieux contaminés non pas par un seul métal mais bien par une multitude de métaux et que d'autre part la physico-chimie des eaux et des sédiments est variable d'un milieu à l'autre. Nos deux rivières présentent effectivement de fortes différences aux points de vue physico-chimique et géochimique. Malgré cela, nous observons des relations significatives entre la concentration de certains métaux mesurés dans les organismes et la concentration de MT. 
La relation entre les métaux et la MT varie d'une espèce à l'autre et d'une rivière à l'autre. Chez l'éphémère $H$. limbata, deux métaux non essentiels, l'Ag et le $\mathrm{Cd}$, ainsi que deux métaux essentiels, le $\mathrm{Ni}$ et le $\mathrm{Zn}$, ont été corrélés positivement à la MT. La relation entre la MT et le $\mathrm{Cd}$ chez les invertébrés a déjà été mentionnée dans quelques travaux (COUILLARD, 1997 ; CROTEAU et al., 2002 ; DEEDS et KLERKS, 1999), et celle avec l'Ag a été observée en laboratoire chez certaines espèces de mollusques (BRAY et al., 1983 ; MOUNEYRAC et al., 2000). Selon VASAK (1991), l'affinité in vitro de la MT pour les métaux est plus forte pour l'Ag que pour le $\mathrm{Cd}[\mathrm{Ag}(\mathrm{I}) \approx \mathrm{Cu}(\mathrm{I})>\mathrm{Cd}(\mathrm{II})>\mathrm{Pb}(\mathrm{II})>\mathrm{Zn}(\mathrm{II})>\mathrm{Ni}(\mathrm{II})]$. II n'est donc pas surprenant d'observer de relations entre la MT et l'Ag et le Cd. D'autre part, la relation entre la MT et le Zn est, semble-t-il, logique car le Zn cytosolique est a priori fixé au moins partiellement à la MT et c'est l'entrée du $\mathrm{Cd}$ qui en déplaçant le $\mathrm{Zn}$ peut déclencher la synthèse de MT (PALMITER, 1994). D'ailleurs, le $\mathrm{Zn}$ ne varie que dans une gamme relativement restreinte de concentrations tissulaires chez les éphémères $\left(190-383 \mu \mathrm{g} \cdot \mathrm{g}^{-1}\right)$ des deux rivières, c'est-à-dire que les rapports Max/Min $(1,7)$ sont faibles par rapport aux variations en [Zn] dissous (rapports Max/Min de 54 et 22). Une relation Zn - MT a d'ailleurs déjà été observée chez les mollusques (BAUDRIMONT et al., 1999 ; BORDIN et al., 1997). Quant à la corrélation négative entre le Cu bioaccumulé et la MT, elle est difficile à expliquer avec les données disponibles. Si la concentration des métaux cystosoliques avait été mesurée au lieu des métaux totaux tissulaires, cela aurait facilité l'interprétation de telles interactions puisque les métaux totaux tissulaires peuvent être liés à d'autres structures (ex. : granules) ou composés que la MT.

Chez le mollusque, le faible nombre de stations dans la rivière Colombière ne permet pas de bien discerner de relation significative entre les différents métaux et la $\mathrm{MT}$, mais il nous semble néanmoins pertinent de mentionner la corrélation avec le $\mathrm{Pb}$. Dans une revue de la littérature sur la MT, COUILLARD (1997) mentionne que des relations entre la MT et le $\mathrm{Pb}$ n'ont jamais été observées chez les mollusques.

Chez le brochet, le $\mathrm{Pb}$ et le $\mathrm{Cd}$ sont significativement et positivement corrélés à la MT. Plusieurs travaux mentionnent des relations entre la concentration de certains métaux (ex. : $\mathrm{Cd}, \mathrm{Cu}, \mathrm{Zn}$ ) et la MT chez plusieurs espèces de poissons (ex.: KLAVERKAMP et al., 1991 ; LAFLAMME et al., 2000 ; RoCH et al., 1982 ; VAN CLEEF et al., 2002) mais aucune à notre connaissance ne fait mention d'une relation positive ou même négative entre le $\mathrm{Pb}$ et la $\mathrm{MT}$. Dans sa revue de la littérature, COUILLARD (1997) montre que ce sont souvent les mêmes métaux qui sont étudiés chez les poissons, soit le $\mathrm{Cd}$, le $\mathrm{Cu}$ et le $\mathrm{Zn}$. Dans notre étude, le $\mathrm{Pb}$ a été corrélé à la MT chez deux de nos trois espèces. Bien que l'affinité in vitro du $\mathrm{Pb}$ avec la MT soit plus faible que pour le $\mathrm{Cd}$ (VASAK, 1991), la relation est tout à fait possible et d'intérêt. Ce résultat mérite d'être approfondi par d'autres études en milieu naturel ou en laboratoire.

\subsection{Le ou les métaux responsables de la synthèse de MT}

Le second objectif de ce travail était de déterminer si les métaux qui induisent la synthèse de la MT différaient entre les espèces. Les résultats de la présente étude sont conformes à ceux de nombreux travaux antérieurs. Ainsi, le cadmium semble être le principal métal déclencheur de la production de MT chez les mollusques (COUILLARD et al., 1993 ; COUILLARD et al., 1995 ; PERCEVAL 
et al., 2002), chez Hexagenia limbata (COUILLARD, 1997) ainsi que chez d'autres invertébrés (CROTEAU et al,, 2002 ; DEEDS et KLERKS, 1999). Le rôle du Cd comme principal initiateur de la synthèse de MT ne semble cependant pas aussi clair chez les poissons. Bien que certains travaux aient démontré de bonnes relations entre le Cd bioaccumulé et la MT (OLSSON et HAUX, 1986 ; KLAVERKAMP et al., 1991; LEUNG et FURNESS, 1999), d'autres études ont démontré le rôle d'autres métaux ( $\mathrm{Cu}, \mathrm{Zn}$ ) comme initiateur de synthèse de MT (HOGSTRAND et HAUX, 1996 ; HOGSTRAND et al., 1989 ; ROCH et al., 1982). Nos travaux et la littérature à ce jour nous indiquent donc que la synthèse de MT peut être associée à différents métaux. COUILLARD (1997) mentionne d'ailleurs que les récentes études tendent à démontrer que le degré d'implication de la MT dans le métabolisme des métaux dépend de l'espèce, de l'organe et du métal en cause. La présente étude démontre que pour les mêmes milieux, pour trois espèces différentes, et pour différents organes (branchies de $P$. grandis, rein de $E$. lucius) ou pour le corps entier chez $H$. limbata, le Cd est le principal initiateur de MT.

\subsection{Relation entre la MT et l'environnement}

Les modèles de régression multiple n'ont pas permis de déceler de facteurs géochimiques ou physico-chimiques susceptibles d'influencer les niveaux de MT chez l'éphémère $H$. limbata. Par contre, la concentration de manganèse dans les sédiments semble influencer négativement la concentration de MT chez le mollusque $P$. grandis. Les oxydes de manganèse sont de bons ligands pour les métaux traces (MURRAY, 1975) et peuvent donc influencer fortement la biodisponibilité du Cd.

\section{5 - CONCLUSION}

Il existe beaucoup de résultats contradictoires dans la littérature au sujet des fonctions biologiques de la MT et des métaux traces susceptibles d'en induire la synthèse. Peu de travaux ont été réalisés en milieu naturel et ce sont souvent les mêmes métaux ou organismes qui font l'objet de ces travaux. De plus, les travaux antérieurs réalisés en milieux lacustres contaminés par plusieurs métaux, dont le gradient de contamination était similaire de lac en lac, ne permettaient pas de différencier l'importance relative des différents métaux sur la synthèse de MT. Cette étude est la première réalisée en rivières, en ciblant trois espèces dans un même milieu récepteur présentant des différences marquées dans la concentration des métaux ambiants entre les stations. Elle indique que le $\mathrm{Cd}$, lorsque présent dans le milieu à des concentrations appréciables, peut initier la synthèse de MT chez des organismes de différents niveaux trophiques présents dans le même milieu. L'étude simultanée de plusieurs espèces, se différenciant par leur mode d'accumulation des métaux, permet donc de mieux comprendre l'impact des métaux sur les processus de synthèse de la MT. En effet, on note que des métaux autres que le cadmium peuvent être impliqués dans ce processus de synthèse, tels que le plomb chez le brochet $E$. lucius et le zinc chez l'éphémère $H$. limbata. 


\section{REMERCIEMENTS}

Ce travail a été rendu possible grâce à une subvention du Conseil de Recherches en Sciences naturelles et en Génie du Canada (CRSNG : programme de partenariats université-industrie) accordée au groupe de recherche dirigé par P.G.C. Campbell. Nous remercions Marie-Claude Tardif, MarieHélène Michaud, Marie-Pier Cloutier et Annick Michaud pour leur aide sur le terrain. Nous remercions également Noranda Inc. (Matagami) et l'Université du Québec en Abitibi Témiscamingue à Val d'Or, qui nous ont permis de réaliser certaines analyses dans leurs laboratoires. Un merci tout spécial à Olivier Perceval pour ses commentaires sur le manuscrit, ainsi qu'aux techniciens et techniciennes de l'INRS-ETE et de COREM pour leur aide lors de l'analyse des différents paramètres physico-chimiques et toxicologiques. Les recherches de P.G.C. Campbell sont appuyées par la chaire de recherche du Canada en écotoxicologie des métaux.

\section{RÉFÉRENCES BIBLIOGRAPHIQUES}

BAUDRIMONT M., ANDRÈS S., METIVAUD J.Y., LAPAQUELLERIE F., RIBEYRE N., MAILLET C., LATOUCHE A., BOUDOU A., 1999. Field transplantation of the freshwater bivalve Corbicula fluminea along a polymetallic contamination gradient (River Lot, France): II. Metallothionein response to metal exposure. Environ. Toxicol. Chem., 18, 2472-2477.

BORDIN G., MCCOURT J., RAPOSO F.C., RODRIGUEZ A. R., 1997. Metallothionein-like metalloproteins in the Baltic clam Macoma balthica: seasonal variations and induction upon metal exposure. Mar. Biol., 129, 453-463.

BRAY J.T., WEBB L.A., REILLY, F.J. Jr., 1983. Multielement analysis of metal-binding proteins in cytosolic fractions. Sci. Tot. Environ., 28, 367-374.

CAMPBELL, P.G.C., COUILLARD Y., 2004. Prise en charge et détoxication des métaux chez les organismes aquatiques. Dans «Écotoxicologie moléculaire ». Eds : E. Pelletier, P.G.C. Campbell et F. Denizeau. Presses de l'Université du Québec, pp. 9-61.

COUILLARD Y., CAMPBELL P.G.C., TESSIER A., 1993. Response of metallothionein concentrations in a freshwater bivalve (Anodonta grandis) along an environmental cadmium gradient. Limnol. Oceanogr., 38, 299-313.

COUILLARD Y., CAMPBELL P.G.C., TESSIER A., PELLERIN-MASSICOTTE J., AUCLAIR J.C., 1995. Field transplantation of a freshwater bivalve, Pyganodon grandis, across a metal contamination gradient. 1. Temporal changes in metallothionein and metal $(\mathrm{Cd}, \mathrm{Cu}$, and $\mathrm{Zn}$ ) concentrations in soft tissues. Can. J. Fish. Aquat. Sci., 52, 690-702.

COUILLARD Y., 1997. Technical evaluation of metallothionein as a biomarker for the mining industry. AETE Project. 2.2.1. Aquatic effects technology. Evaluation Program. Ressources naturelles Canada. $191 \mathrm{p}$.

CROTEAU M-N., HARE L., CAMPBELL P.G.C., COUILLARD Y., 2002. Metallothionein-like metal-binding protein in the biomonitor Chaoborus; occurrence and relationship to metal concentrations in lakes. Environ. Toxicol. Chem., 21, 737741.

DEEDS J.R., KLERKS P.L., 1999. Metallothionein-like proteins in the freshwater oligochaete Limnodrilus udekemianus and their role as a homeostatic mecha- 
nism against cadmium toxicity. Environ. Pollut, 106, 381-389.

FARAG A.M., STANSBURY M.A., HOGSTRAND C., MACCONNELL E., BERGMAN H.L., 1995. Physiological impairment of free-ranging brown trout in the Clark Fork River, Montana. Can. J. Fish. Aquat. Sci, 52, 2038-2050.

FARAG A.M., SKAAR D., NIMICK D.A., MACCONNELL E., HOGSTRAND C., 2003. Characterizing aquatic health using salmonid mortality, physiology, and biomass estimates in streams with elevated concentrations of arsenic, cadmium, copper, lead, and zinc in the Boulder river watershed, Montana. Trans. Amer. Fish. Soc., 132, 450-467.

HARE, L. 1992. Aquatic insects and trace metals: bioavailability, bioaccumulation, and toxicity. Crit. Rev. Toxicol., 22, 327369.

HOGSTRAND C., HAUX C., 1996. Naturally high levels of zinc and metallothionein in liver of several squirellfish families from Queensland, Australia. Mar. Biol., 125, 23-31.

HOGSTRAND C., LITHNER G., HAUX C., 1989. Relationship between metallothionein, copper and zinc in perch (Perca fluviatilis) environmentally exposed to heavy metals. Mar. Environ. Res., 28, 179-182.

KÄGI J.H.R., KOJIMA Y., 1987. Chemistry and biochemistry of metallothioneins. Dans « Metallothionein II », KÄGI J.H.R. et KOJIMA Y. [Eds], Experientia Suppl., 52, 25-61.

KLAVERKAMP J.F., DUTTON M.D., MAJEWSKI H.S., HUNT R.V., WESSON L.J., 1991. Evaluating the effectiveness of metal pollution controls in a smelter by using metallothionein and other biochemical responses in fish. Dans « Metal ecotoxicology - Concepts and Applications ", NEWMAN M.C. et MCINTOSH A.W. [Eds], Lewis Publishers Inc., Chelsea, MI, USA, pp. 33-64.

LAFLAMME J.-S., COUILLARD Y., CAMPBELL P.G.C., HONTELA A., 2000. Interrenal metallothionein and cortisol secretion in relation to $\mathrm{Cd}, \mathrm{Cu}$, and $\mathrm{Zn}$ exposure in yellow perch, Perca flavescens, from Abitibi lakes. Can. J. Fish. Aquat. Sci., 57, 1692-1700.
LEGENDRE P., LEGENDRE, L., 1998. Numerical Ecology, 2nd edition. Elsevier, Amsterdam.

LEUNG K.M.Y., FURNESS R.W., 1999. Induction of metallothionein in dogwhelk Nucella lapillus during and after exposure to cadmium. Ecotox. Env. Safety, 43, 156-164.

MCINTOSH, A.W., 1991. Trace metals in freshwater sediments: a review of the literature and an assessment of research needs. Dans « Metal Ecotoxicology, Concepts and Applications ", NEWMAN M.C. et MCINTOSH A.W. [Eds], Lewis Publishers Inc., Chelsea, MI, USA, pp. 243-260.

MOUNEYRAC C., GEFFARD A., AMIARD J.C., AMIARD-TRIQUET C., 2000. Metallothionein-like proteins in Macoma balthica: effect of metal exposure and natural factors. Can. J. Fish. Aquat. Sci, 57, 34-42.

MURRAY J.W., 1975. Interaction of metal ions at manganese dioxide-solution interface. Geochim. Cosmochim. Acta, 39, 505-519.

NRIAGU J.O., 1990. Global metal pollution. Poisoning the biosphere. Environment (Wash. DC), 32, 7-33.

OLSSON P.-E., HAUX C., 1986. Increased hepatic metallothionein content correlates to cadmium accumulation in environmentally exposed perch (Perca fluviatilis). Aquat. Toxicol., 9, 231-242.

OLSVIK P.A., GUNDERSEN P., ANDERSEN R.A., ZACHARIASSEN K.E., 2000. Metal accumulation and metallothionein in two populations of brown trout, Salmo trutta, exposed to different natural water environments during a run-off episode. Aquat. Toxicol., 50, 301-316.

OLSVIK P.A., HINDAR K., ZACHARIASSEN K.E., ANDERSEN R.A., 2001. Brown trout (Salmo trutta) metallothioneins as biomarkers for metal exposure in two Norwegian rivers. Biomarkers, 6, 274288.

PALMITER R.D., 1994. Regulation of metallothionein genes by heavy metals appears to be mediated by a zinc-sensitive inhibitor that interacts with a constitutively active transcription factor, MTF-1. Proc. Natl. Acad. Sci. USA, 91, 1219-1223. 
PERCEVAL O., PINEL-ALLOUL B., MÉTHOT G., COUILLARD Y., GIGUÈRE A., CAMPBELL P.G.C., HARE L., 2002. Cadmium accumulation and metallothionein synthesis in freshwater bivalves (Pyganodon grandis): relative influence of the metal exposure gradient versus limnological variability. Environ. Pollut., 118, 5-17.

ROCH M., MCCARTER J.A., MATHERSON A.T., CLARK M.J.R., OLAFSON R.W., 1982. Hepatic metallothionein in rainbow trout (Salmo gairdnerii) as an indicator of metal pollution in the Campbell River system. Can. J. Fish. Aquat. Sci., 39, 1596-1601.

ROESIJADI G., 1980. The significance of low molecular weight, metallothionein-like proteins in marine invertebrates: current status. Mar. Environ. Res., 4, 167-179.

ROESIJADI G., 1996. Metallothionein and its role in toxic metal regulation. Comp. Biochem. Physiol., 113C, 117-123.
SMITH D.A., 1987. Mining in America. University Press of Kansas, Lawrence, KS, USA.

VAN CLEEF K.A., KAPLAN L.A.E., CRIVELLO J.F., 2002. The relationship between reproductive status and metallothionein mRNA expression in the common killifish, Fundulus heteroclitus. Envir. Biol. Fish., 57, 97-105.

VASAK M., 1991. Metal removal and substitution in vertebrate and invertebrate metallothioneins. Dans "Methods in Enzymology ", ABELSON J.N. et SIMON M.I. [Eds], Academic Press Inc., San Diego, CA, USA, pp. 452-458.

VIARENGO A., 1989. Heavy metals in marine invertebrates: mechanisms of regulation and toxicity at the cellular level. CRC Crit. Rev. Aquat. Sci., 1, 295-317.

VIARENGO A., BURLANDO B., DONDERO F., MARRO A., FABBRI R., 1999. Metallothionein as a tool in biomonitoring programmes. Biomarkers, 4, 455-466. 